\title{
Hepatotoxicity Following Immune Checkpoint Inhibitor Therapy in Metastatic Lung Adenocarcinoma: A Case Report
}

\section{Shike Lou}

Shanghai Jiao Tong University Medical School Affiliated Ruijin Hospital

\section{Xiaolin Wang}

Shanghai Jiao Tong University Medical School Affiliated Ruijin Hospital

\section{Fei Yuan}

Shanghai Jiao Tong University Medical School Affiliated Ruijin Hospital

\section{Gangde Zhao}

Shanghai Jiao Tong University Medical School Affiliated Ruijin Hospital

\section{Lanyi Lin}

Shanghai Jiao Tong University Medical School Affiliated Ruijin Hospital

\section{Yezhou Ding}

Shanghai Jiao Tong University Medical School Affiliated Ruijin Hospital

Kehui Liu

Shanghai Jiao Tong University Medical School Affiliated Ruijin Hospital

\section{Qing Xie}

Shanghai Jiao Tong University Medical School Affiliated Ruijin Hospital

\section{Shisan Bao}

Shanghai Jiao Tong University Medical School Affiliated Ruijin Hospital https://orcid.org/0000-00026687-3846

\section{Hui Wang ( $\nabla$ wanghuirj@163.com )}

Shanghai Jiao Tong University Medical School Affiliated Ruijin Hospital https://orcid.org/0000-00021440-8942

\section{Research Article}

Keywords: Immune checkpoint inhibitors, lung adenocarcinoma, hepatotoxicity, liver biopsy

Posted Date: February 7th, 2022

DOI: https://doi.org/10.21203/rs.3.rs-1224141/v1 
License: (c) (i) This work is licensed under a Creative Commons Attribution 4.0 International License. Read Full License 


\section{Abstract}

Immune checkpoint inhibitors (ICls) are mAbs against cytotoxic T-lymphocyte antigen 4 (CTLA-4), programmed death-1 receptor (PD-1) and programmed death-ligand 1 (PD-L1), which the inhibitory pathways that leads to T cell inactivation. Here, we report a case of ICl-induced immune-mediated hepatotoxicity with liver biopsy. A 63-year-old man with brain metastasis from lung adenocarcinoma was treated with three times of $\mathrm{g}$ knife treatment, following by a combination of chemotherapy [carboplatin and (550 mg) + pemetrexed (1000 mg)] plus bevacizumab (anti-VEGF monoclonal antibody) (600 mg D1) and camrelizumab (anti-PD-1 mAb) (200 mg D1). Around 21 days later, abnormal liver function was detected. He received a diagnosis of ICl-induced liver injury after liver biopsy. The liver function of this patient was recovered, following methylprednisolone treatment. Such data offers useful information for clinicians to deal with cancer patients for ICl treatment, suggesting that liver function must be closely monitored.

\section{Introduction}

Immune checkpoint inhibitors (ICl) has been wildly used for anti-cancer therapy in various malignancies [1], however, the outcomes of $\mathrm{ICl}$ have been compromised in some degree among a number of patients with serious immune-related adverse events (irAE) [2], including skin, gastrointestinal tract, endocrine glands and liver. Hepatotoxicity may be one of the most dangerous complications with $\mathrm{ICl}$, occurring in up to $16 \%$ of patients receiving ICls [3]. However it was not fully demonstrated the case of ICl-induced immune-mediated hepatotoxicity from the literature. We reported here a case of ICI-induced immunemediated hepatotoxicity with liver biopsy.

\section{Case}

A 63-year patient was admitted to neurosurgery for neurological symptoms due to lung adenocarcinoma brain metastasis. Histopathology of his tumor showed PD-L1 tumor proportion score (TPS) $20 \%$ and KRAS mutation in Exon2, but EGFR mutations and ALK rearrangements were negative. This patient was discharged on the same day following a combination of chemotherapy [carboplatin and (550 mg) + pemetrexed (1000 mg)] plus bevacizumab (anti-VEGF monoclonal antibody) (600 mg D1) and camrelizumab (anti-PD-1 mAb) (immune check point inhibitor) (200 mg D1). However on the third day, this patient had maculopapular rash on the chest with itching and tongue ulcers, but was resolved spontaneously 3 days later. Abnormal liver function was discovered during his second admission for the anti-cancer therapy, showing that ALT, AST, ALP or GGT was 268IU/L, $280 \mathrm{IU} / \mathrm{L}, 259 \mathrm{IU} / \mathrm{L}$ or $344 \mathrm{IU} / \mathrm{L}$, respectively; TB or DB was 12.7 or $3.9 \mu \mathrm{mol} / \mathrm{L}$, respectively (Figure1). He had no viral hepatitis nor autoimmune liver disease. $B$ ultrasound and magnetic resonance revealed a normal liver and bile duct structure. Notably, DILI susceptible gene screened from this patient showed a RS72631567 mutation with RUCAM score at 6. 
Liver biopsy of this patient demonstrated that focal necrosis, acidophilic body and mild steatosis and cholestasis (Figure 2). Lymphocyte, but not eosinophil infiltration, was observed primarily in the liver sinusoids and mild inflammation in the portal area. These infiltrating lymphocytes were $\mathrm{CD}^{+}$or $\mathrm{CD} 8^{+}$, but not CD20+. CK19 immuno-staining showed normal small bile ducts. HBsAg, HbcAg, Copper and Perls blue were all negative.

Thus the cause of the liver injury was finally diagnosed as ICl-induced immune-mediated hepatotoxicity at level 3, according to the Common Adverse Reaction Toxicity Criteria (CTCAE 4.03 criteria) from National Cancer Institute [4]. ICI was discontinued and corticosteroid was initiated. The liver function of this patient was recovered, following methylprednisolone treatment. Finally, the patient's liver function almost recovered, showing that ALT, AST, ALP or GGT was 44, 32, 326 or $367 \mathrm{IU} / \mathrm{L}$, respectively, prior to discharge (Figure 3).

\section{Discussion}

ICl-induced immune-mediated hepatotoxicity is manifested as elevated AST and ALT or symptomatic hepatitis [5], and $>50 \%$ of the cases are cholestatic hepatitis [6], then mixed liver injury are more common than hepatocellular liver injury [7].

In the current case, the patient has received pemetrexed and carboplatin initially, followed by anti-PD1. Thus, it is rather challenging for the clinicians to clarify whether such liver injury was caused by pemetrexed and carboplatin and/or PD-1. This patient presented with chest maculopapular with itching and tongue ulcer, more favorable in $\mathrm{ICl}$ induced liver injury, which is supported with liver biopsy of the histopathology. We can't exclude the liver injury from this patient caused by paclitaxel and carboplatin, which often resulted in more severe liver damage at histopathological level, i.e. fusion necrosis and bile duct damage [8]. Our finding, however, is more favorable to $\mathrm{ICl}$ induced liver damage, showing that quite large number of $\mathrm{CD}^{+}$or $\mathrm{CD}^{+}$lymphocyte, but not eosinophils, infiltration in the damaged liver, which is in agreement with other report of $\mathrm{ICl}$ injured liver [9][10]. Some researchers further showed that portal vein inflammatory cells are composed of $\mathrm{CD} 4^{+}$and $\mathrm{CD} 8^{+} \mathrm{T}$ cells, and the lobules are dominated by $\mathrm{CD} 8^{+}$ cells[5]. In this case, the histological morphology is manifested by punctate necrosis of hepatocytes and inflammation in the portal area, accompanied by infiltration of $\mathrm{CD} 4^{+}$and $\mathrm{CD} 8^{+} \mathrm{T}$ cells. These histological characteristics are consistent with ICI injured liver.

\section{Summary}

Here, we reported a case of ICl induced liver injury, with clinical manifestations and pathology. The pathology demonstrated that this patient included focal necrosis, acidophilic body, mild steatosis, mild cholestasis and portal inflammation, as well as infiltrating leucocytes in portal area, mainly $\mathrm{CD}^{+}$and CD8 ${ }^{+}$lymphocytes. This ICI induced liver injury patient responded well to corticosteroid. Such data offers useful information for clinicians to deal with cancer patients with ICI treatment, suggesting that liver function must be closely monitored. 


\section{Declarations}

\section{Acknowledgements}

The authors thank the patient for his participation in this study and for the patients' agreement to the publication of the report.

\section{Authors' contributions}

S.K.L, K.H.L, and Y.Z.D conceived of the case and collected data, S.K.L , X.L.W and F.Y drafted the manuscript. G.D.Z , L.Y.L and Q.X assisted with manuscript writing, editing and detailed search of references. H.W and S.S.B as the co-corresponding authors, were involved in the study design and revision of the draft and all authors commented on previous versions of the manuscript. All authors read and approved the final manuscript.

\section{Funding}

The authors declare that no funds, grants, or other support were received during the preparation of this manuscript.

\section{Data availability}

All related datas are presented in this paper, further information or datas are available on a reasonable request.

\section{Code availability}

Not applicable.

\section{Compliance with ethical standards}

\section{Ethics approval}

The case report comply with the current laws of the country in which he was performed.

\section{Consent to participate}

The patient declared to consent to participate in this study.

\section{Consent for publication}

The patient declared to consent to the publication of the current paper.

\section{Conflicts of interest}

The authors declare that they have no conflict of interest. 


\section{Research involving human participants}

This work was performed in humans with approval from the Institutional Review Board.

\section{Informed consent}

The Institutional Review Board granted a waiver for this retrospective study.

\section{References}

1. Benitez JC, Remon J, Besse B (2020) Current Panorama and Challenges for Neoadjuvant Cancer Immunotherapy. Clin Cancer Res 26:5068-5077. https://doi.org/10.1158/1078-0432.CCR-19-3255

2. Sosa A, Cadena EL, Olive CS et al (2018) Clinical assessment of immune-related adverse events. Ther Adv Med Oncol 10:1-11. https://doi.org/10.1177/1758835918764628

3. Peeraphatdit T, Wang J, Odenwald MA et al (eds) (2020) Hepatotoxicity From Immune Checkpoint Inhibitors: A Systematic Review and Management Recommendation. Hepatology 72:315-329. https://doi.org/10.1002/hep.31227

4. Health UD, Services H (2016) others Common terminology criteria for adverse events (CTCAE) version 4.03. 2010. USA Natl Inst Health Natl Cancer Inst

5. Martin ED (2018) Characterization of liver injury induced by cancer immunotherapy using immune checkpoint inhibitors. J Hepatol 68:1181-1190. https://doi.org/10.1016/j.jhep.2018.01.033

6. Mizuno K, Ito T, Ishigami M et al (2020) Real world data of liver injury induced by immune checkpoint inhibitors in Japanese patients with advanced malignancies. J Gastroenterol 55:653-661. https://doi.org/10.1007/s00535-020-01677-9

7. Imoto K, Kohjima M, Hioki T et al (2019) Clinical Features of Liver Injury Induced by Immune Checkpoint Inhibitors in Japanese Patients. Can J Gastroenterol Hepatol 2019:1-12. https://doi.org/10.1155/2019/6391712

8. Kleiner DE, Chalasani NP, Lee WM et al (2014) Hepatic histological findings in suspected druginduced liver injury: Systematic evaluation and clinical associations. Hepatology 59:661-670. https://doi.org/10.1002/hep.26709

9. Zen Y, Yeh MM (2018) Hepatotoxicity of immune checkpoint inhibitors: a histology study of seven cases in comparison with autoimmune hepatitis and idiosyncratic drug-induced liver injury. Mod Pathol 31:965-973. https://doi.org/10.1038/s41379-018-0013-y

10. Zen $Y$, Chen $Y$, Jeng $Y$ et al (2020) Immune-related adverse reactions in the hepatobiliary system: second-generation check-point inhibitors highlight diverse histological changes. Histopathology 76:470-480. https://doi.org/10.1111/his. 14000

\section{Figures}




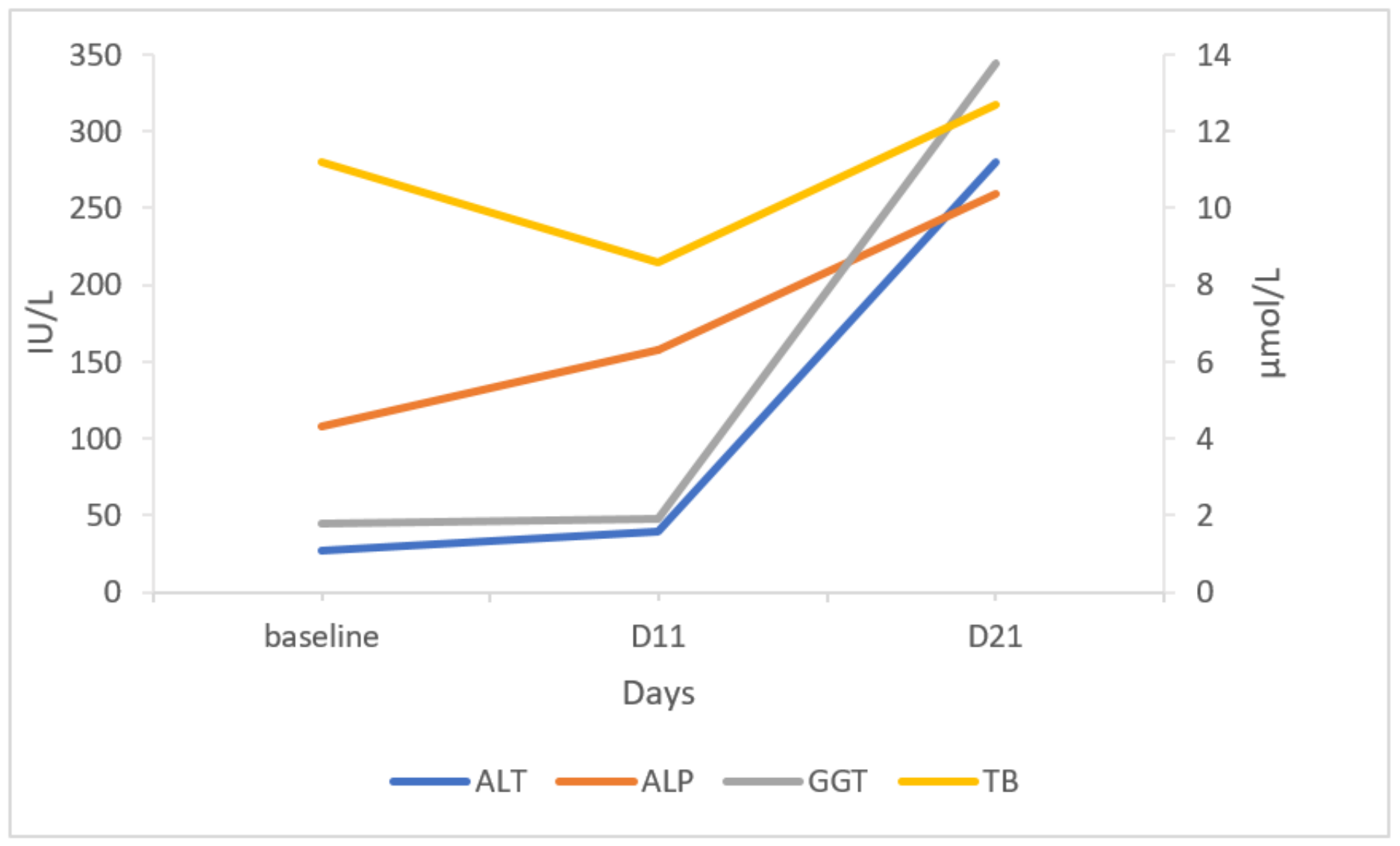

Figure 1

The changes of specific indicators of liver function before and after immunotherapy. 

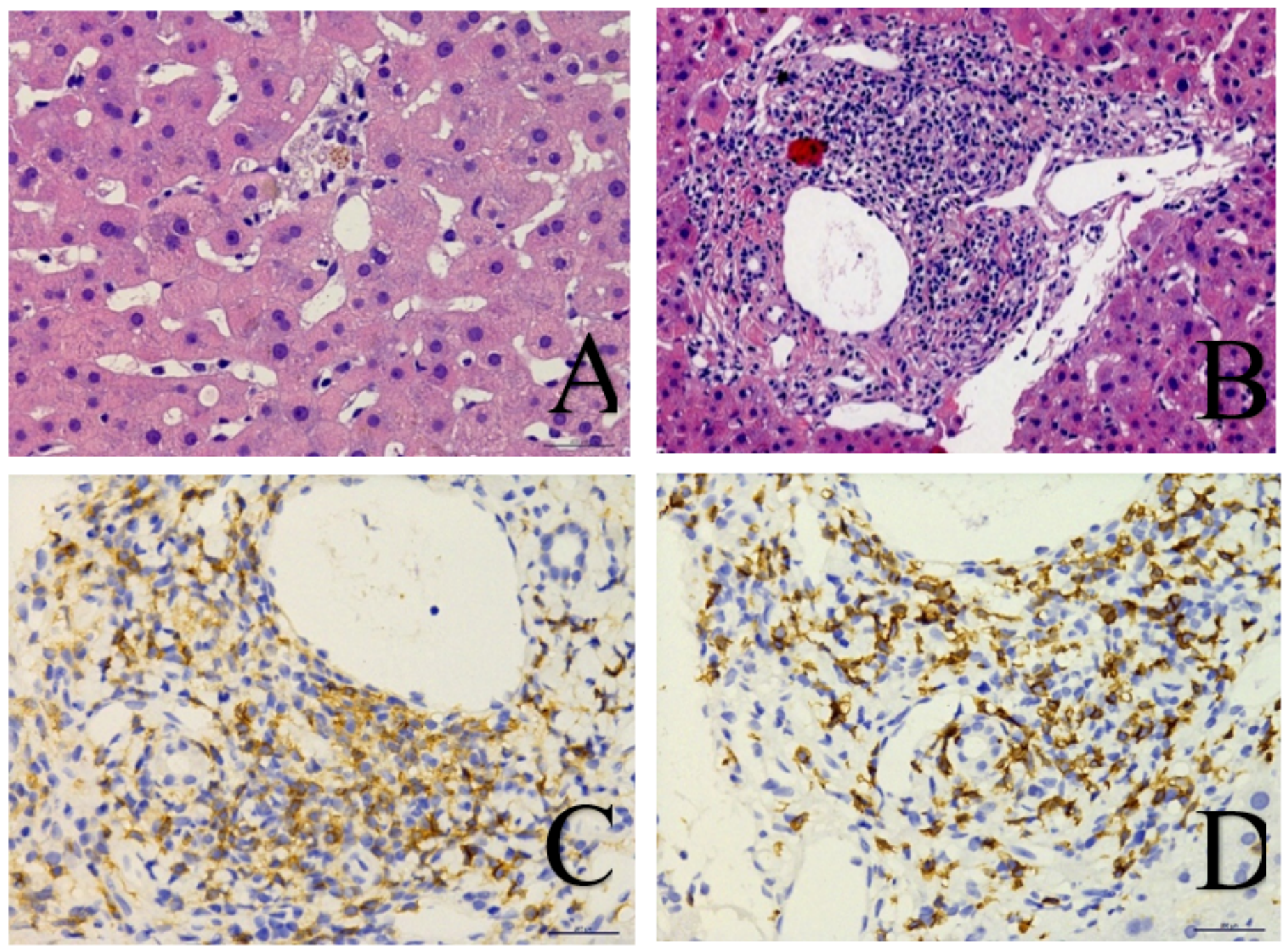

\section{Figure 2}

Liver biopsy showed spotted focal necrosis (Figure A) infiltrated inflammatory cells, mainly lymphocytes in portal area (Figure B), and the enrichment of CD4 (Figure C) and CD8 (Figure D) in portal area. 


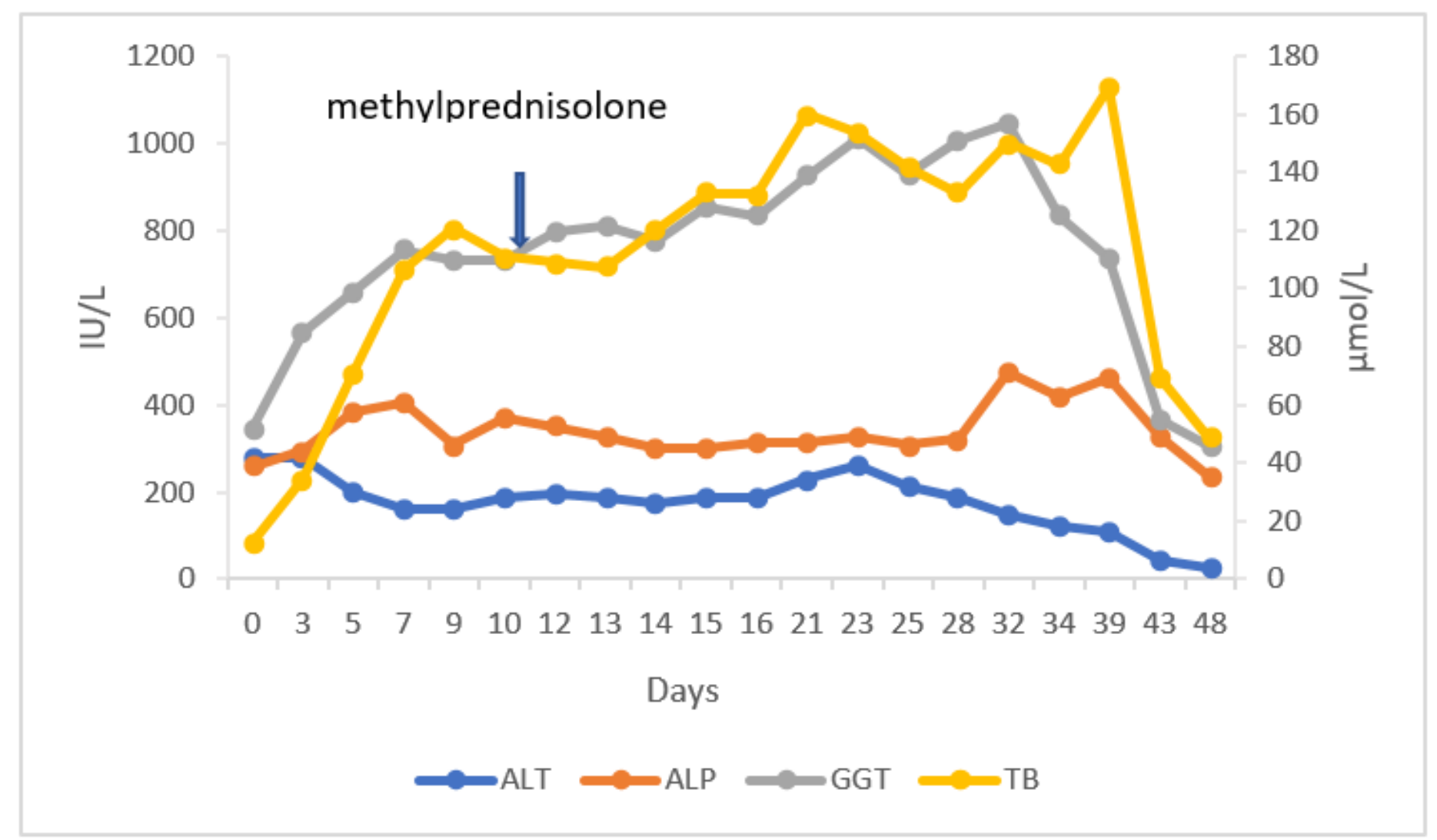

Figure 3

The changes of specific indicators of liver function before and after methylprednisolone treatment. 